Voix et Images

voixetimages

\title{
La littérature montréalaise et les ghettos
}

\section{Benoît Melançon}

Volume 16, numéro 3 (48), printemps 1991

François Charron

URI : https://id.erudit.org/iderudit/200923ar

DOI : https://doi.org/10.7202/200923ar

Aller au sommaire du numéro

Éditeur(s)

Université du Québec à Montréal

ISSN

0318-9201 (imprimé)

1705-933X (numérique)

Découvrir la revue

Citer cet article

Melançon, B. (1991). La littérature montréalaise et les ghettos. Voix et Images, 16(3), 482-492. https://doi.org/10.7202/200923ar d'utilisation que vous pouvez consulter en ligne.

https://apropos.erudit.org/fr/usagers/politique-dutilisation/ 


\section{La littérature montréalaise et les ghettos}

\section{par Benoît Melançon, Université de Montréal}

Dans les Promenades littéraires dans Montréal qu'ils viennent de faire paraître, Monique LaRue et Jean-François Chassay ont choisi d'inaugurer leur parcours par un chapitre intitulé "Ville de villages et de villageois ". 1 . Si ce titre laisse entendre que l'intégration des Montréalais à leur ville s'est faite sur le modèle d'un monde à dominance rurale, il indique également un aspect de la constitution imaginaire de Montréal qui rallie un grand nombre de créateurs et de critiques littéraires: son image dans la littérature repose sur une forte ségrégation des espaces. La difficulté à saisir Montréal dans sa globalité, écrit Yannick Resch, s'explique par le fait que Montréal est composée de zones où s'affrontent des ethnies, où s'exaspèrent des tensions, où s'opposent des comportements sociaux ${ }^{2}$. Les termes pour exprimer cette ségrégation sont nombreux: quartier, d'abord, qui domine le champ sémantique; village ou faubourg, pour reprendre des expressions de Bonheur d'occasion de Gabrielle Roy (1947); petite patrie, chez Claude Jasmin ${ }^{3}$. Un autre terme est présent dans les textes sur Montréal, mais on ne s'est jamais interrogé sur le rôle qu'il pouvait jouer dans la littérature montréalaise: il s'agit du mot ghetto 4 . Afin de voir sur quel horizon se découpe le champ sémantique de ce mot, nous voudrions livrer quelques réflexions préliminaires sur cette question à partir de trois types d'exemples: la description du ghetto juif des

1 Monique LaRue, en collaboration avec Jean-François Chassay, Promenades littéraires dans Montréal, Montréal, Québec/Amérique, 1989, 274 p.

2 Yannick Resch, "Limaginaire de la ville: Montréal dans la fiction québécoise de 1940 à 1980 », thèse de doctorat d'État, Université de Provence (Aix-Marseille I), 1985, p. 375. Voir aussi p. 147-149.

3 Le romancier de la Petite Patrie (1972) raconte: Nous étions élevés comme dans le temps des villes fermées et emmurées du moyen áge. Le quartier était un village, un monde clos. [...] Aussi, tout ce qui ne nous ressemblait pas était sujet de méfiance (cité par Monique LaRue et Jean-François Chassay, op. cit., p. 20). Voir aussi l'ouvrage récent de François Hébert, Montréal: Montréal a éclaté, s'est dispersé à l'intérieur de soi-même, est devenu la somme de ses fragments, une marguerite de petites patries (Seyssel, Champ Vallon, 1989, p. 97 [Des villes]).

4 L'analyse ne portera que sur les ghettos de Montreal. On pourrait cependant étudier les ghettos d'Europe de l'Est dans des textes d'écrivains québécois: Chava Rosenfarb (en yiddish), Alice Parizeau (en français), etc. 
années cinquante chez Yves Thériault et Mordecai Richler, le sens du mot ghetto chez les écrivains francophones dans les années soixante et son utilisation par un dramaturge d'origine italienne, Marco Micone. Ces réflexions préliminaires s'inscrivent dans le cadre de recherches menées à l'Université de Montréal sur les représentations littéraires de Montréal et, plus particulièrement, sur la ville imaginaire des communautés culturelles ${ }^{5}$. Avant d'aborder les trois corpus, deux remarques s'imposent sur les raisons qui justifient le choix de cet objet.

La première de ces remarques touche directement le domaine de la littérature. Depuis de nombreuses années, le mot ghetto a été utilisé pour dire la réalité spatiale montréalaise; c'est vrai des créateurs comme des critiques. D'une part, l'on ne s'étonne guère de voir sous la plume des écrivains des allusions au ghetto juif de la rue Saint-Laurent ou à ce que des générations d'étudiants ont appelé le ghetto de McGill (autour de l'université du même nom). Chez David Fennario, le ghetto de VerdunPointe Saint-Charles ${ }^{6}$ se rapproche, par sa situation économique, des ghettos juif ou étudiant. On s'étonnera en revanche de voir certains parler du Golden Ghetto of Outremont ${ }^{7}$ ou assimiler Westmount ${ }^{8}$ et Notre-Dame-de-Grâce ${ }^{9}$ à des ghettos. D'autre part, la critique a aussi utilisé le mot pour décrire des espaces qui ne se présentent pas explicitement comme tels. Le mot ghetto, par exemple, n'apparait jamais dans Bonheur d'occasion, comme permet de le vérifier la concordance du roman ${ }^{10}$. Or, on trouve sous la plume de Marjorie Fitzpatrick, de Robert Mane et de Yannick Resch le mot ghetto au sujet de SaintHenri ${ }^{11}$. Il ne s'agit évidemment pas de reprocher aux́ critiques l'usage du mot, mais de constater sa fortune et de s'interroger sur elle.

La seconde remarque est plus sociologique que littéraire. Pas une semaine ne se passe actuellement à Montréal sans que les journaux ne

5 On consultera Benoît Melançon, la Littérature montréalaise des communautés culturelles. Prolégomènes et bibliographie, Montréal, Université de Montréal, Département d'études françaises, Groupe de recherche Montréal imaginaire, mars 1990, $31 \mathrm{p}$.

6 Que l'écrivain distingue du student ghetto de la rue Aylmer (Blue Mondays, Verdun, Black Rock Creations, 1984, p. 13). Selon Jean-Claude Marsan, il n'est pas exagéré de parler de Pointe Saint-Charles comme du ghetto du Grand-Tronc (Montrëal. Une esquisse du futur, Québec, IQRC, 1983, p. 45).

7 Herman Buller, One Man Alone, Toronto, Canada National Book Club. Canadiana, 1963, p. 288.

8 Edward Phillips, Sunday's Child, Toronto, McClelland and Stewart, 1981, p. 15.

9 Témoignage d'Arnold Bennett recueilli par Allen Gottheil, les Juifs progressistes au Québec, Montréal, Éditions par ailleurs..., 1988, p. 243.

10 Paul G. Socken, Concordance de Bonheur d'occasion de Gabrielle Roy, Waterloo, University of Waterloo Press, 1982, vii-1135 p.

11 Marjorie A. Fitzpatrick, "Teaching French-Canadian Civilization Through the Literature: Hémon, Roy, and Blais „, Québec Studies, 2, 1984, p. 85, 87; Yannick Resch, "Montréal dans l'imaginaire des écrivains québécois", Études canadiennes / Canadian Studies, 19, décembre 1985, p. 140; Robert Mane, “L'image de Montréal dans les Chroniques du Plateau Mont-Royal ", ibid., p. 119, 129. 
fassent allusion aux divers ghettos qui menaceraient le tissu urbain. Plusieurs faits divers récents, dont certains tragiques, ont attiré l'attention sur la question de l'intégration des immigrants dans la société montréalaise. Les médias, lorsqu'ils traitent cette question, mettent régulièrement leurs lecteurs en garde contre les dangers de la ghettoïsation: Comment débarrasser Montréal de ses ghettos?, s'interrogeait par exemple Pierre Vennat, éditorialiste au quotidien la Presse, le 3 juillet 1989. Le recours au champ sémantique du ghetto ne se limite cependant pas aux réflexions sur les immigrants de fraîche date. L'élection des quatre députés anglophones du Parti Égalité aux élections provinciales de septembre a ainsi été interprétée par certains comme la manifestation d'une ghettoïsation de la communauté angloquébécoise ${ }^{12}$. S'il est vrai que l'on peut expliquer cette contamination des discours par la richesse métaphorique du mot ghetto - ne parle-ton pas de ghettos d'emploi ou d'écoles-ghettos ${ }^{13}$ ? -, il n'en reste pas moins vrai que son apparition dans le discours social ne peut manquer, et de plus en plus, au fil et à mesure du développement de l'immigration au Québec, de faire sentir ses effets dans les discours littéraires.

Le seul ghetto auquel se sont intéressés de façon soutenue les critiques jusqu'à maintenant est le ghetto juif ${ }^{14}$. Plusieurs raisons expliquent cet intérêt. La première est que le ghetto - faut-il le rappeler ? - est étymologiquement et historiquement lié à la culture juivé. À cet égard, sa présence dans le Montréal littéraire n'a rien d'étonnant - mais encore faut-il souligner que ce ghetto a ses caractéristiques propres. Une deuxième raison est liée au corpus: c'est sur ce ghetto qu'il existe le plus grand nombre de textes littéraires. Du côté francophone - et pour ne prendre que des exemples romanesques -, Yves Thériault ${ }^{15}$, Pierre Gélinas ${ }^{16}$, Monique Bosco ${ }^{17}$ et

12 Bref, les anglophones risquent encore plus de se retrouver dans un ghetto, écrit Alain Dubuc ("La victoire d'Égalité: un signe d'immaturité politique», la Presse, 28 septembre 1989, p. B2). Voir aussi Claude Masson, "Un heureux dénouement", la Presse, 26 septembre 1989, p. B2.

13 Méme le discours sportif est touché: selon Réjean Tremblay, Ile Québec est le ghetto du baseball à cause de la langue ("Dawson le "choker" ", la Presse, 11 octobre 1989 , p. S13). A la limite, tout peut relever du ghetto: pour les personnages de Lux de Pierre Filion (Montréal, Leméac, 1989), l'informatique, c'est rendu une forme d'idéologie, c'est un véritable ghetto cérébral (p. 20). Ou encore cette phrase sur les hommes: Entre eux, dans leur ghetto sauce modernite, rien ne pouvait les atteindre et les compromettre (p. 123).

14. Voir Antoine Sirois, Montréal dans le roman canadien, Montréal, Marcel Didier, 1968, p. 59-65, 107-111, 125-127, 137-139; Yannick Resch, "Le "ghetto" juif ", thèse citee, p. 363-380; Michael Greenstein, "Beyond the Ghetto and the Garrison: Jewish-Canadian Boundaries», Mosaic, 14, 2, printemps 1981, p. 121-130.

15 Yves Thériault, Aaron (1954 et 1957), Montréal, Quinze, 1981, 157 p. (10/10).

16 Pierre Gélinas, les Vivants, les morts et les autres, Montréal, CLF, 1959, 314 p. Selon le narrateur, les traditions et coutumes du ghetto jurent avec la quiétude de la vie canadienne (cité par Antoine Sirois, op. cit., p. 126).

17 Monique Bosco, Un amour maladroit, Paris, Gallimard, 1961, 213 p. L'auteure parle de ghetto malodorant et fétide (cité par Antoine Sirois, op. cit., p. 36). 
Andrée Maillet ${ }^{18}$ ont, à des degrés divers, écrit sur ce ghetto. Du côté anglophone, les textes de Saul Bellow ${ }^{19}$, d'A.M. Klein ${ }^{20}$, de Sholem Shtern ${ }^{21}$ et, surtout, l'ensemble de l'œuvre romanesque de Mordecai Richler ont fait des rues entourant le boulevard Saint-Laurent un des hauts lieux 'de la littérature montréalaise. Une troisième raison peut expliquer la fortune critique du ghetto juif: il semble en effet possible de proposer une hypothèse - qui reste à développer - selon laquelle la constitution de ce ghetto, puis la migration de ses habitants hors de ses murs (métaphoriques), pourrait servir de modèle à une réflexion sur la migration intra-urbaine de tous les Montréalais, cette migration étant à la fois physique et économique.

- Deux romans serviront à expliciter notre propos. Paru d'abord en 1954, puis repris et modifié en 1957, Aaron d'Yves Thériault est le récit de la lutte que se livrent le jeune Aaron Cashin et son grand-père Moishe au sujet de la place des Juifs dans la société matérialiste nordaméricaine. On trouve dans Son of a Smaller Hero (en français: Mon père, ce héros... ${ }^{22}$ ) de Mordecai Richler, publié pour la première fois en 1955, une intrigue qui n'est pas sans rappeler celle du roman de Thériault: comme Aaron, Noah Adler est déchiré entre l'orthodoxie religieuse, symbolisée par la figure du grand-père, et le monde moderne, qui est aussi celui de la femme. Chez Thériault, le personnage féminin est une Juive d'origine française, Viedna, alors que, chez Richler; Miriam a été élevée à Montréal dans le quartier de Griffintown; dans les deux cas, elles représentent tout à la fois la modernité et l'extérieur du ghetto, ce ghetto en train, sinon de mourir, du moins d'éclater ${ }^{23}$. Mais le ghetto des deux romanciers est-il identique?

18 Andrée Maillet, Nouvelles montréalaises, Montréal, Librairie Beauchemin, 1966, 132 p.; repris sous le titre les Montréalais, Montréal, l'Hexagone, 1987, 338 p. (Typo, 13).

19 Saul Bellow, Dangling Man, New York, 1975. Michael Greenstein étudie ce roman (art. cité, p. 122-123).

20 Voir les premières pages de "A Myriad-Minded Man", dans Short Stories, édition de M.W. Steinberg, Toronto, University of Toronto Press, 1983, p. 125-153 (coll. Collected Works of A. M. Klein, 2).

21 , Sholem Shtern, The Household of Professor Sydney Goldstein. A Novel in Verse/La Maisonnée du professeur Sydney Goldstein. Un roman en vers, Montréal, [s.é.], 1984, 101 p. Édition bilingue.

22 Mordecai Richler, Mon père, ce héros..., Montréal, CLF, 1975, 350 p. (Collection des deux solitudes). Traduction de Jean Simard.

23 Peut-il mourir? Y retournant vingt ans après la publication de son roman "Chez "Aaron" (dans' Robert Guy Scully (édit.), Morceaux du grand Montréal, Longueuil, Éditions du Noroit, 1978, p. 133-136), Thériault montrera que le ghetto, abandonné depuis par les Juifs, est toujours là, mais que ce n'est plus tout à fait lui: Peut-être le quartier est-il encore un ghetto, mais le sens est impropre. Il y a la même pauvreté résignée, mais les odeurs, tout aussi puissantes, ne sont plus les mêmes. [...] Les couleurs ont changé aussi (p. 136). Voir aussi Mordecai Richler, Rue Saint-Urbain; Montréal, HMH, 1969, p. 21-23 (LAArbre, 16). Traduction de Jean Simard. 
Deux traits caractérisent le ghetto de Thériault. Il n'est tout d'abord qu'un ghetto parmi d'autres. Moishe, èn effet, ne s'est installé à Montréal qu'après avoir quitté deux autres ghettos: le ghetto emmuraillé (p. 21) de Minsk, d'où il a été chassé avec sa femme Sarah, puis le quartier sémite (p. 15) de San Francisco, qu'il a quitté avec son fils et son petit-fils après la mort de sa, femme. L'établissement à Montréal n'est donc, pour Moishe, qu'un autre déracinement, qu'une autre migration: c'est là, laisse entendre le grand-père, le sort des Juifs que de toujours être chassés d'un ghetto vers un autre, de remplacer les anciens ghettos (p. 61) par de nouveaux. Par ailleurs, la représentation de Montréal ne s'organise dans le roman qu'autour de deux pôles: le ghetto, que le narrateur enserre dans le quadrilatère formé par les rues Saint-Laurent, Mont-Royal, Saint-Denis et Sherbrooke (p. 26), et le mont Royal, seule possibilité d'évasion pour Aaron. C'est là, dans la nature et non pas à la ville, qu'il fera la connaissance de Viedna ${ }^{24}$. Montréal n'a d'existence, dans le roman, que par le ghetto, les autres lieux évoqués étant fort rares: la place d'Armes, où travaille Aaron après son départ du ghetto, et des banlieues (p. 110) et autres quartiers luxueux (p. 136) à la géographie incertaine. Comme le note Yannick Resch,

le fait que Montréal soit peu décrit est symbolique d'un comportement qui reste en deçà d'une appropriation de la ville. Aaron n'en est encore qu'à rêver de se perdre dans la ville. Il lui reste à la conquérir. ${ }^{25}$

Le ghetto, chez Yves Thériault, est le dernier refuge de l'orthodoxie religieuse ${ }^{26}$ avant d'être une réalité urbaine.

Dans Mon père, ce héros... de Richler, la ségrégation spatiale est beaucoup plus présente que dans le roman de Thériault et témoigne d'un rapport différent à la ville littéraire; le ghetto relève d'un autre modèle d'intelligibilité de la ville. Ainsi de la description de trois quartiers. Celle du ghetto juif, d'abord, qui, même s'il ne comporte ni vrais murs ni vraies dimensions, n'en a pas moins une définition précise: ses franges, selon Richler, sont des rues nommées SaintUrbain, Saint-Dominique, Rachel et de l'Hôtel-de-Ville; son aorte est la rue Saint-Laurent; si on longe cette rue vers le sud, ce n'est plus le ghetto (p. 19); les rues de celui-ci se distinguent nettement des autres rues (à Outremont ou à Snowdon): chaque rue avait sa propre façon de déambuler, son style particulier. Un style si révélateur, que toute violation de territoire sautait aussitôt aux yeux (p. 23) ${ }^{27}$; le ghetto a

24 Sur le mont Royal comme nature, voir p. 62-63.

25 Yannick Resch, thèse citée, p. 369-370.

26 Ce qu'il est aussi: On lui avait raconté [à Aaron] que dans d'autres quartiers de Montréal la vie ne serait pas la même, que s'il traversait en zone défendue on lui jetterait la pierre s'il se disait Juif (p. 31).

27. Et ce, bien qu'il règne dans ces diverses rues le même uesprit de ghettow dont parle Richler dans The Apprenticeship of Duddy Kravitz (cité par Antoine 
ses dictons, éternels, souligne le narrateur (p. 43), et son climat propre: Louis Berger, le bookmaker, affirma qu'il faisait toujours plus chaud de dix degrés dans le ghetto que n'importe où ailleurs dans la ville (p. 236). Il n'en va pas autrement des autres espaces cadastrés dans le roman.

Le "centre-ville», écrit Richler, cela veut généralement dire la rue Sainte-Catherine. Plus précisément, le centre-ville de Montréal épouse la forme d'un rectangle. Borné à l'ouest par la rue Atwater, à l'est par le boulevard Saint-Laurent; au nord et au sud, respectivement, par les rues Sherbrooke et Craig. (p. 37)

Griffintown, troisième quartier aux frontières précises, est pour le romancièr un dédale [...] entre les quais et la rue Wellington (p. 172). Le ghetto, pour Richler, n'est donc pas que le lieu de l'orthodoxie religieuse; c'est, véritablement, une façon de lire la ville et, plus largement, tout espace: les échappées hors de la ville, en l'occurrence dans les Laurentides ${ }^{28}$, sont en effet soumises à une volonté de délimitation de nature semblable (voir le "Chapitre deuxième" du roman).

Ainsi, Montréal n'est pas pour Richler cette chose anonyme faite de deux lieux antithétiques qu'elle était pour Thériault: elle est une réalité à lire dans la différence des espaces, et c'est cette différence qui permet de comprendre la migration dans la ville. S'il serait oiseux de superposer le Westmount de Bonheur d'occasion à l'Outremont de Mon père, ce héros..., ou Saint-Henri au ghetto de Richler, il importe néanmoins de penser la question des ghettos dans leur relation à l'ensemble du tissu urbain. L'expérience du ghetto est indissociable de la ségrégation affichée dans les œuvres. C'est par rapport aux autres quartiers, aux autres petites patries ou aux autres ghettos, que doivent se définir les ghettos juifs montréalais. Ce que disent chacun à leur façon Thériault et Richler, c'est que le ghetto, même s'il se rêve autarcique, ne peut vivre dans la ville moderne sans entrer en contact avec les autres espaces, qu'ils soient naturels (chez Thériault) ou construits (chez Richler). Moishe et Melech, les grands-pères des deux romans, ne peuvent qu'assister, impuissants malgré leur volonté de résistance, à l'effilochage du ghetto. Le ghetto juif est d'abord affaire d'espace, et d'espace menacé.

Si le champ sémantique du ghetto a surtout servi à caractériser à ce jour l'appropriation juive de la ville, on en retrouve toutefois des manifestations dans des discours tout à fait différents de ceux évoqués jusqu'ici. Le modèle dominant du ghetto comme enclave

Sirois, op. cit., p. 59). Sur les caractéristiques différentes de chacune des rues du ghetto, on verra Rue Saint-Urbain, p. 36-37.

28. Shulamis Yelin en parle comme du Jewish summer ghetto (Shulamis. Stories from a Montreal Childhood, Montréal, Vehicule Press, 1983, p. 36). 
juive coexiste, surtout dans les années soixante, avec une lecture non plus religieuse et spatiale du ghetto, mais avec une lecture économique. Parce que le ghetto dans les textes de cette époque est lié à l'exploitation économique, et que ce thème a dominé une bonne partie de la littérature québécoise des années soixante, il oblige la critique à s'interroger sur l'intertexte qui les alimente. On prendra pour premier exemple la description que fait Pierre Vallières des Canadiens français exploités dans Nègres blancs d'Amẹ́rique (1969):

Ces nègres, qui n'ont pas tous la peau de la même couleur, qui ne parlent pas tous la même langue, qui croient en des prophètes différents, qui habitent des ghettos étrangers les uns aux autres et qui subissent de diverses façons la dicta. ture du même système économique, politique et social, tous ces nègres que les esclavagistes, les businessmen et les politiciens s'ingénient, depuis des siècles, à dresser les uns contre les autres pour mieux les exploiter et les maintenir dans l'impuissance, savent, aujourd'hui, que la liberté et la paix, dans ce monde d'argent, de violence et d'oppression, ne peuvent se conquérir que par la force du nombre et des armes. 29

Les nègres blancs dont Vallières se fait le porte-parole partagent des ghettos avec les autres opprimés. Ces ghettos sont le lieu de la pauvreté et de l'exploitation, mais c'est d'eux que sortira cette "révolution globale" dont se réclame Vallières.

Nègres blancs d'Amérique date de 1969. En 1965, on trouvait déjà dans un roman de Jacques Godbout, le Couteau sur la table, une remarque sur les ghettos, encore une fois dans une perspective économique. Le personnage principal déclare à sa maîtresse canadienne-anglaise, Patricia - mais la déclaration est entre guillemets, comme s'il s'agissait d'une citation: "À l'ouest dans la montagne un ghetto monstrueux où des châteaux réservés aux seigneurs d'Albion dominent cette ville qu'un million d'esclaves français, de leur sang..." À cette description ironique de Westmount, Patricia répond: Don't be ridiculous! ${ }^{30}$ Au-delà de l'inversion des signes chez Vallières, les ghettos sont peuplés d'exploités, alors que chez Godbout il s'agit des exploiteurs -, il serait intéressant de retracer l'origine de cette référence au ghetto dans les discours idéologiques des années soixante. Deux sources sont ici à considérer : les tenants du Black Power qui ont œuvré au sein des ghettos noirs des grandes villes américaines (leur rapport à l'œuvre de Vallières se passe de

29 Cité par Laurent Mailhot, avec la collaboration de Benoît Melançon, Essais québécois 1837-1983. Anthologie littéraire, Montréal, Hurtubise HMH, 1984, p. 537-538 (Cahiers du Québec. Textes et documents littéraires, 79). Nous soulignons.

30 Jacques Godbout, le Couteau sur la table, Paris, Seuil, 1965, p. 88-89. 
commentaire) et les théoriciens de la décolonisation (dont Albert Memmi, Juif tunisien, qui a vécu l'expérience du ghetto, la "hara", dans son pays d'origine). Ces deux * sources ", proposées à titre d'hypothèses, devraient servir à comprendre le champ sémantique du ghetto dans la littérature francophone des années soixante. La gestion des espaces, si importante dans le cas du ghetto juif de Thériault et de Richler, aurait toujours un rôle à jouer, mais ce rôle serait subordonné à une réflexion économique et, par extension, politique. Les murs du ghetto - s'ils existent - ne seraient pas ceux de la religion mais ceux du capital. De cette conception, on pourra trouver la forme extrême chez le Pierre Vadeboncœur de la Dernière Heure et la première en 1970. Pour l'essayiste, la ville - toute ville - est un ghetto, parce que son développement coïncide avec
la fin précipitée, récente et presqu'achevée [sic] de la propriété rurale du pays par les masses, [...] la domination de la propriété industrielle et commerciale (étrangère) sur la pro- priété agricole (autochtone), le déclin de notre supériorité démographique, la désintégration de notre culture originelle, la corruption croissante de notre langue. ${ }^{31}$

Si l'horizon sur lequel se détache la position de Vadeboncœur a peu à voir idéologiquement avec les projets révolutionnaires de Vallières et l'ironie de Godbout, elle participe du même discours social.

La question de l'intégration des communautés à la ville, qui s'est posée à des moments divers pour les Juifs et les Québécois francophones, reste douloureusement d'actualité pour les Montréalais d'immigration récente. Le théâtre de Marco Micone est révélateur à cet égard. Bien que le mot ghetto n'apparaisse que dans la première pièce de Micone, Gens du silence (1982) ${ }^{32}$, la réalité qu'il désigne se fait encore sentir, mais de façon plus discrète, dans Addolorata (1984) et Déjà l'agonie (1988): le quartier italien de la première pièce, que l'auteur appelle Chiuso ("fermé" en italien) et qu'il associe explicitement au ghetto, est remplacé par l'image de l'immeuble à étages dans la deuxième pièce ${ }^{33}$, puis par celle des villages étriqués et méprisés,

31 Pierre Vadeboncour, la Dernière Heure et la première, Montréal, l'Hexagone et Parti pris, 1970, p. 50.

32 Deux occurrences du mot sont particulièrement intéressantes, car elles indiquent comment se créent les ghettos. Dans la première - Il faut prendre d'autres moyens que les gardiens du ghetto n'ont pas encore pris (Gens du silence, Montréal, Québec/Amérique, 1982, p. 94 [Premières]) -, les gardiens dont il est question font partie de la communauté italienne elle-même. Dans la seconde On a jamais voulu de nous. Ils nous refusaient même leurs écoles. C'est pour ç̧a que Chiuso existe. C'est pour ça que les immigrants ont leurs quartiers, qu'ils ont, précise un autre personnage, leurs écoles-ghettos (ibid., p. 135) -, le ils désigne les Francesi (ibid., p. 114). La première position est celle de la militante Nancy, et la seconde, celle de son pere conservateur, Antonio.

33 Marco Micone, Déjà l'agonie, Montréal, Guernica, 1984, p. 9-11 et 43-44. 
dans la troisième ${ }^{34}$. Enfermé entre trois carrières de ciment et le Boulevard Métropolitain, Chiuso est, comme Saint-Henri ${ }^{35}$, le lieu d'une oppression économique au bénéfice du Haut-Montréal ${ }^{36}$. Il partagerait donc des traits avec le ghetto juif (l'enfermement géographique) et avec le ghetto économique dénoncé dans les années soixante.

La coupure des immigrants italiens avec la société québécoise francophone et anglophone est le thème central des pièces de Micone, où elle est doublement métaphorisée. Économique, elle repose sur ce que Micone appelle la culture immigrée ${ }^{37}$ : Si l'émigration était une bonne chose, on l'aurait pas laissée aux pauvres comme nous autres et nos parents, écrit-il dans Addolorata (p. 49) ${ }^{38}$. C'est pour des raisons de main-d'œuvre - docilité forcée, faiblesse des coûts - que le Québec s'est ouvert à l'immigration italienne. Il n'a jamais eu intérêt, soutient le dramaturge, à faire sortir les Italiens de cette forme d'oppression. Géographique, la coupure est de telle nature que l'immigrant italien vit toujours dans des ghettos, et ce, malgré l'ascension sociale. Une scène d'Addolorata rend bien l'impossibilité pour l'immigrant de sortir de sa communauté. Par un jeu d'alternances qui rappelle le théâtre de Michel Tremblay, le personnage d'Addolorata envoie, à dix ans d'intervalle, des invitations à son mariage et des cartes de remerciement à la suite de la mort de sa mère. Chaque nom de personnage est suivi dans un premier temps de

34 "Hors-texte», dans Déjà l'agonie, Montréal, l'Hexagone, 1988, p. 15 (Thếtre). La préfacière, Monique LaRue, souligne l'importance de la coexistence de ces espaces qui échouent d̀ se compénétrer vraiment (ibid., p. 13).

35 Notre pays, celui de Chiuso et de Saint-Henri, est à faire, dit le chœur qui clót la piece (Gens du silence, op. čit., p. 140).

36 Gens du silence, op. cit., p. 55. On trouve le même découpage géographique dans Addolorata (p. 62), où le quartier est, de plus; opposé à d'autres lieux: avant.de faire le tour du monde, Addolorata aimerait pouvoir aller en bas de la ville, au moins une fois, danser dans une vraie discotheque (p. 25).

37 D'inspiration brechtienne, le théatre de Micone propose de faire éclater toutes les limites du ghetto: Il faut remplacer la culture du silence par la culture immigrée, dit un des personnages de Gens du silence, pour que le paysan en nous se redresse, pour que l'immigrant en nous se souvienne et pour que le Québécois en nous commence d̀ vivre. Je ne ferai plus le mouton dans un enclos (p. 95). Le personnage de Nancy reprend à ce moment une position défendue plus tót dans la pièce: Je me libère de Chiuso pour mieux parler de liberté (p. 92), alors que pour son père, Chiuso est le plus beau quartier de Montréal (p. 115).

38 Plusieurs de ses autres déclarations vont dans ce sens. Présentant un extrait d'Addolorata (dans Fulvio Caccia et Antonio D'Alfonso (éditeurs), Quêtes. Textes d'auteurs italo-québécois, Montréal, Guernica, 1983, p. 27), il soutient que Il'émigration n'aurait jamais existé si elle avait pu aider à l'émancipation de tous ceux qui furent emportés par elle. Dans une entrevue accordée à Fulvio Caccia, il déclare: Le ghetto existe, et il va exister pendant longtemps pour deux raisons: premièrement, parce qu'il y a des individus qui travaillent à sa perpétuation et, deuxiemement, parce qu'il y en a d'autres qui croient qu'il n'existe pas (Sous le signe du Phénix. Entretiens avec quinze créateurs italoquébécois, Montréal, Guernica, 1985, p. 266). 
son ancienne adresse, puis est répété, suivi de sa nouvelle. Le grégarisme de la communauté italienne est souligné par ce procédé: pour qui connaît la géographie montréalaise, il est clair que les ItaloQuébécois n'ont quitté un quartier que pour mieux se rassembler ailleurs. Cette fausse diaspora, Micone la souligne quand il insère, dans l'alternance des adresses, un personnage qui, lui, n'a pas déménagé. Le nom de ce personnage, Pasquale Stabile («stable" en français), montre que la mobilité sociale reste factice si elle ne s'accompagne pas d'un éclatement des murs de la communauté ${ }^{39}$.

Pour reprendre des catégories que proposait récemment Pierre Nepveu pour définir les divers types de ce que l'on appelle la «transculture", on pourrait dire de Micone, que Nepveu donne d'ailleurs en exemple, que ses pièces relèvent d'une transculture vécue comme aliénation, perte de soi, dérive ${ }^{40}$. L'attitude de Micone se distinguerait, toujours selon la classification de Nepveu, d'une littérature immigrante vue comme expérience de la mémoire ${ }^{41}$ et d'une expérience de repérage [...] tributaire d'un brouillage sémantique et géographique ${ }^{42}$. On peut supposer que Micone, par son insistance sur la dimension économique du ghetto et sur l'échec de la sortie hors de ses murs, puise dans deux types de discours sur la réalité urbaine et tient, précisément à cause de cette réflexion sur le ghetto, une place à part dans la littérature immigrante.

La pratique de Micone ne le distingue pas uniquement des écrivains immigrants, mais aussi de plusieurs écrivains québécois "de souche" (à défaut de meilleure expression). L'expérience du ghetto, telle que décrite sous ses divers aspects par Micone, est en effet complètement distincte des succès romanesques récents dans lesquels l'espace est livré à la mobilité, pour citer Gilles Marcotte ${ }^{43}$. Il y a lieu de s'interroger sur l'existence, dans la production immédiatement contemporaine, de textes dénonçant l'enfermement, ceux de

39 Addolorata, p. 20-21.

40 "Qu'est-ce que la transculture? ", Paragraphes, 2, 1989, p. 21.

41 Ibid., p. 22. Nepveu donne Marilú Mallet en exemple.

42 Ibid., p. 23. Nepveu donne la Québécoite (1983) de Régine Robin en exemple. Cette interprétation rejoint celle que donne Yannick Resch du roman (thèse citée, p. 391-392) et celle de Simon Harel du Piano-trompette (1983) de Jean Basile ("Les marges de la ville: identité et cosmopolitisme dans le roman montréalais", dans Gilles Marcotte (éditeur), Lire Montréal. Actes du Colloque tenu le 21 octobre 1988 à l'Université de Montréal, Montréal, Université de Montréal, Département d'études françaises, Groupe de recherche Montréal imaginaire, 1989, p. 21-36).

43 "Le temps du Matou ", Paragraphes, 2, 1989, p. 44. On comparera cette lecture des romans de Michel Tremblay, entre autres, à celle de Ginette Michaud ("Mille plateaux: topographie et typographie d'un quartier *, dans Gilles Marcotte (editeur), op. cit., p. 38-71): l'auteure parle du Plateau Mont-Royal comme d'une enceinte quasi sacrée qui assimile presque alors le quartier au ghetto (p. 62) et de quartier-ghetto (p. 68). 
Micone, par exemple, et de textes pour lesquels les frontières urbaines explosent, chez Yolande Villemaire ou Nicole. Brossard 44 . L'expérience du ghetto ne pourrait-elle pas expliquer en partie le rapport à la ville de certains écrivains immigrants? Leur arrivée en ville ne rappelle-t-elle pas celle des francophones? L'expérience du ghetto des écrivains immigrants n'a-t-elle pas alors quelque chose à dire sur le rapport de ces francophones à la ville?

On remarquera que n'ont pas été prises ici en considération les frontières entre les genres, pas plus que la positivité qui peut parfois être associée au ghetto comme image de la force du lien communautaire ou comme mode d'accès à la ville ${ }^{45}$. Il paraissait nécessaire dans un premier temps d'émettre un certain nombre d'hypothèses sur la nature des ghettos dans la littérature montréalaise. Celles-ci pourraient être résumées ainsi : le ghetto juif, celui qui a été le plus largement étudié jusqu'à maintenant, doit être compris dans le contexte de la ségrégation montréalaise des espaces et peut servir de point de départ à une réflexion sur la migration urbaine (malgré les apparences; la religion et la mobilité sociale n'y seraient peut-être pas les facteurs déterminants); le ghetto des écrivains francophones des années soixante, lui, renvoie à une pensée économique qui s'appuie sans aucun doute sur cette ségrégation, mais pour laquelle elle n'a pas le rôle central qu'elle a dans le cadre du ghetto juif; le ghetto vécu par les immigrants de fraîche date, enfin, tout en se situant par rapport aux deux premiers types de ghetto, pose le problème de l'insertion de leurs textes dans les courants dominants de la transculture et de la littérature québécoise. Ces hypothèses devront être relues, et confirmées ou infirmées, à la lumière d'autres textes du corpus montréalais. Peut-être pourra-t-on alors mieux penser l'insertion, toujours à faire, de l'Autre dans le tissu urbain.

44 Ma ville est multiple. Je ne sais pas mettre de clotures entre les quartiers, les parkings étalés du centre-ville. [...] Je sais seulement marcher entre les saisons, la tête haute, le regard neuf comme si Montréal était une aventure toujours à recommencer (Nicole, Brossard, "Aura d'une ville", dans Montréal des écrivains, Montréal, l'Hexagone et Únion des écrivains québécois, 1988, p. 38 [Typo, 31]).

45 L'exhaustivité n'a pas non plus été visée. Il aurait fallu parler des rares présences du ghetto noir - chez Morley Callaghan (The Loved and the Lost, 1951) ou Gilles Lamer (Bâtissez mon temple..., 1985). De plus, les mécanismes de la constitution des ghettos (sont-ils imposés à l'Autre, ou n'est-ce pas plutốt l'Autre qui s'y refugie de son propre gre ?) et les questions linguistiques (déterminantes chez Micone) ont été volontairement passés sous silence. 